\title{
Generalized Quasi-Variational Inequalities for Pseudo- Monotone Type III and Strongly Pseudo-Monotone Type III Operators on Non-Compact Sets
}

\author{
Mohammad S. R. Chowdhury ${ }^{1, ~}$, Yeol Je Cho ${ }^{2,3}$ \\ ${ }^{1}$ Department of Mathematics, University of Management and Technology, Lahore, Pakistan \\ ${ }^{2}$ Department of Mathematics Education and RINS, Gyeongsang National University, Jinju, Korea \\ ${ }^{3}$ Department of Mathematics, King Abdulaziz University, Jeddah, Saudi Arabia \\ Email address: \\ msrchowdhury@hotmail.com (M. S. R. Chowdhury), showkat.chowdhury@umt.edu.pk (M. S. R. Chowdhury), \\ yjchomath@gmail.com (Y. J. Cho)
}

\section{To cite this article:}

Mohammad S. R. Chowdhury, Yeol Je Cho. Generalized Quasi-Variational Inequalities for Pseudo-Monotone Type III and Strongly PseudoMonotone Type III Operators on Non-Compact Sets. American Journal of Applied Mathematics. Special Issue: Proceedings of the 1st UMT National Conference on Pure and Applied Mathematics (1st UNCPAM 2015). Vol. 3, No. 3-1, 2015, pp. 46-53.

doi: $10.11648 /$ j.ajam.s.2015030301.18

\begin{abstract}
In this paper, the authors prove some existence results of solutions for a new class of generalized quasi-variational inequalities (GQVI) for pseudo-monotone type III operators and strongly pseudo-monotone type III operators defined on noncompact sets in locally convex Hausdorff topological vector spaces. In obtaining these results on GQVI for pseudo-monotone type III operators, we shall use Chowdhury and Tan's generalized version [1] of Ky Fan's minimax inequality [2] as the main tool.
\end{abstract}

Keywords: Generalized Quasi-Variational Inequalities, Pseudo-Monotone Type III Operators,

Locally Convex Topological Vector Spaces

\section{Introduction}

Let $X$ be a non-empty set, and $2^{X}$ be the family of all nonempty subsets of $X$. Let $E$ be a topological vector space. We shall denote by $E^{*}$ the continuous dual of $E$, by $\langle w, x\rangle$ the pairing between $E^{*}$ and $E$ for $w \in E^{*}$ and $x \in E$ and by $\operatorname{Re}\langle w, x\rangle$ the real part of $\langle w, x\rangle$. Given the maps $S: X \rightarrow 2^{X}$ and $T: X \rightarrow 2^{E^{*}}$, the generalized quasi-variational inequality problem (GQVI) is to find a point $\hat{y} \in S(\hat{y})$ and a point $\widehat{w} \in T(\hat{y})$ such that $\operatorname{Re}\langle\widehat{w}, \hat{y}-x\rangle \leq 0$ for all $x \in S(\hat{y})$. The GQVI was introduced by Chan and Pang [3] in 1982 when $E$ is finite dimensional and by Shih and Tan [4] in 1985 when $E$ is infinite dimensional.

In [5] we established some existence theorems of generalized variational inequalities and generalized complementarity problems in topological vector spaces for pseudo-monotone type III operators defined as follows:

Definition 1.1. Let $E$ be a topological vector space, $X$ a non-empty subset of $E$ and $T: X \rightarrow 2^{E^{*}}$ a map. If $h: X \rightarrow \mathbb{R}$, then $T$ is said to be an h-pseudo-monotone (respectively, a strongly h-pseudo-monotone) type III operator if for each $x, y \in X$ and every net $\left\{y_{\alpha}\right\}_{\alpha \in \Gamma}$ in $X$ converging to $y$ (respectively, weakly to $y$ ) with

$$
\lim \sup _{\alpha \in \Gamma}\left[\inf _{u \in T\left(y_{\alpha}\right)} \operatorname{Re}\left\langle u, y_{\alpha}-x\right\rangle+h\left(y_{\alpha}\right)-h(x)\right] \leq 0,
$$

we have

$$
\begin{aligned}
\limsup _{\alpha \in \Gamma}\left[\inf _{u \in T\left(y_{\alpha}\right)}\right. & \left.\operatorname{Re}\left\langle u, y_{\alpha}-y\right\rangle+h\left(y_{\alpha}\right)-h(y)\right] \\
& \geq \inf _{w \in T(y)} \operatorname{Re}\langle w, y-x\rangle+h(y)-h(x) .
\end{aligned}
$$

$T$ is said to be a pseudo-monotone (respectively, a strongly pseudo-monotone) type III operator if $\mathrm{T}$ is an h-pseudomonotone type III (respectively, a strongly h-pseudomonotone type III) operator with $h \equiv 0$.

The above operators were originally named $h$-hemicontinuous (respectively, strong $h$-hemi-continuous) operators in [5]. Later, in [6], we re-named these operators pseudo-monotone type III operators.

The following result in [5] justified the validity of a setvalued pseudo-monotone (respectively, strongly pseudomonotone) type III operator. 
Proposition 1.1. Let $X$ be a non-empty compact subset of a topological vector space $E$ and $T: X \rightarrow 2^{E^{*}}$ an upper semicontinuous mapping from the relative weak topology on $X$ to the strong topology on $E^{*}$, such that each $T(x)$ is a strongly compact subset of $E^{*}$. Then $T$ is both a pseudo-monotone and a strongly pseudo-monotone type III operator.

If $T$ is single-valued and continuous, the compactness of $X$ is not required and the following result was obtained in [5]:

Proposition 1.2. Let $X$ be a non-empty bounded subset of a topological vector space $E$ and $T: X \rightarrow E^{*}$ a continuous mapping from the relative weak topology on $X$ to the strong topology on $E^{*}$. Then $T$ is both a pseudo-monotone and a strongly pseudo-monotone type III operator.

In this paper, we shall first obtain some general theorems on solutions for a new class of generalized quasi-variational inequalities for pseudo-monotone type III operators and strongly pseudo-monotone type III operators defined on noncompact sets in topological vector spaces. In obtaining these results, we shall mainly use the following generalized version of Ky Fan's minimax inequality [2] due to M.S.R. Chowdhury and K.-K Tan [1].

Theorem 1.3. Let $E$ be a topological vector space, $X$ be a non-empty convex subset of $E, h: X \rightarrow \mathbb{R}$ be lower semicontinuous on $\operatorname{co}(A)$ for each $A \in \mathcal{F}(X)$, and $f: X \times X \rightarrow$ $\mathbb{R} \cup\{-\infty,+\infty\}$ be such that

(a) for each $A \in \mathcal{F}(X)$ and each fixed $x \in \operatorname{co}(A), y \mapsto$ $f(x, y)$ is lower semi-continuous on $\operatorname{co}(A)$;

(b) for each $A \in \mathcal{F}(X)$ and each $y \in \operatorname{co}(A), \min _{x \in A}[f(x, y)+$ $h(y)-h(x)] \leq 0$

(c) for each $A \in \mathcal{F}(X)$ and each $x, y \in \operatorname{co}(A)$, every net $\left\{y_{\alpha}\right\}_{\alpha \in \Gamma}$ in $X$ converging to $y$ with $f(t x+$ $\left.(1-t) y, y_{\alpha}\right)+h\left(y_{\alpha}\right)-h(t x+(1-t) y) \leq 0$ for all $\alpha \in \Gamma$ and all $t \in[0,1]$, we have $f(x, y)+h(y)-$ $h(x) \leq 0$;

(d) there exist a non-empty closed and compact subset $K$ of $X$ and $x_{0} \in K$ such that $f\left(x_{0}, y\right)+h(y)-h\left(x_{0}\right)>0$ for all $y \in X \backslash K$.

Then there exists $\hat{y} \in K$ such that $f(x, \hat{y})+h(\hat{y})-$ $h(x) \leq 0$ for all $x \in X$.

\section{Preliminaries}

Let $E$ be a topological vector space over $\Phi$. Then, for each $x_{0} \in E$, each non-empty subset $A$ of $E$ and each $\epsilon>0$, let $W\left(x_{0} ; \epsilon\right):=\left\{y \in E^{*}:\left|\left\langle y, x_{0}\right\rangle\right|<\epsilon\right\} \quad$ and $U(A ; \epsilon)$ $:=\left\{y \in E^{*}: \sup _{x \in A}|\langle y, x\rangle|<\epsilon\right\}$.

Let $\sigma\left\langle E^{*}, E\right\rangle$ be the topology on $E^{*}$ generated by the family $\{W(x ; \epsilon): x \in E$ and $\epsilon>0\}$ as a subbase for the neighborhood system at 0 and $\delta\left\langle E^{*}, E\right\rangle$ be the topology on $E^{*}$ generated by the family $\{U(A ; \epsilon): A$ is a non-empty bounded subset of $E$ and $\epsilon>0\}$ as a base for the neighborhood system at 0 . We note that $E^{*}$, when equipped with the topology $\sigma\left\langle E^{*}, E\right\rangle$ or the topology $\delta\left\langle E^{*}, E\right\rangle$, becomes a locally convex Hausdorff topological vector space. Furthermore, for a net $\left\{y_{\alpha}\right\}_{\alpha \in \Gamma}$ in $E^{*}$ and for $y \in E^{*}$, (i) $y_{\alpha} \rightarrow y$ in $\sigma\left\langle E^{*}, E\right\rangle$ if and only if $\left\langle y_{\alpha}, x\right\rangle \rightarrow\langle y, x\rangle$ for each $x \in E$ and (ii) $y_{\alpha} \rightarrow y$ in $\delta\left\langle E^{*}, E\right\rangle$ if and only if $\left\langle y_{\alpha}, x\right\rangle \rightarrow\langle y, x\rangle$ uniformly for $x \in A$ for each non-empty bounded subset $A$ of $E$. The topology $\sigma\left\langle E^{*}, E\right\rangle$ (respectively, $\delta\left\langle E^{*}, E\right\rangle$ ) is called the weak*topology (respectively, the strong topology) on $E^{*}$.

If $X$ is a topological space and $\left\{U_{\alpha}: \alpha \in \mathcal{A}\right\}$ is an open cover for $X$, then a partition of unity subordinated to the open cover $\left\{U_{\alpha}: \alpha \in \mathcal{A}\right\}$ is a family $\left\{\beta_{\alpha}: \alpha \in \mathcal{A}\right\}$ of continuous real-valued functions $\beta_{\alpha}: X \rightarrow[0,1]$ such that

(a) $\beta_{\alpha}(y)=0$ for all $y \in X \backslash U_{\alpha}$,

(b) $\left\{\right.$ support $\left.\beta_{\alpha}: \alpha \in \mathcal{A}\right\}$ is locally finite and

(c) $\sum_{\alpha \in \mathcal{A}} \beta_{\alpha}(y)=1$ for each $y \in X$.

We shall first state the following result which is Lemma 1 of Shih and Tan in [4, pp.334-335]:

Lemma 2.1. Let $X$ be a non-empty subset of a Hausdorff topological vector space $E$ and $S: X \rightarrow 2^{E}$ be an upper semicontinuous map such that $S(x)$ is a bounded subset of $E$ for each $x \in X$. Then for each continuous linear functional $p$ on $E$, the map $f_{p}: X \rightarrow \mathbb{R}$ defined by $f_{p}(y)=\sup _{x \in S(y)} \operatorname{Re}\langle p, x\rangle$ is upper semi-continuous; i.e. for each $\lambda \in \mathbb{R}$, the set $\left\{y \in X: f_{p}(y)=\sup _{x \in S(y)} \operatorname{Re}\langle p, x\rangle<\lambda\right\}$ is open in $X$.

The following result is Lemma 3 of Takahashi in [7, p.177] (see also Lemma 3 in [8, pp.68-85]):

Lemma 2.2. Let $X$ and $Y$ be topological spaces, $f: X \rightarrow \mathbb{R}$ be non-negative and continuous and $g: Y \rightarrow \mathbb{R}$ be lower semi-continuous. Then the map $F: X \times Y \rightarrow \mathbb{R}$, defined by $F(x, y)=f(x) g(y)$ for all $(x, y) \in X \times Y$, is lower semicontinuous.

We shall need the following Kneser's minimax theorem in [9, pp.2418-2420] (see also [10, pp.40-41]):

Theorem 2.3. Let $X$ be a non-empty convex subset of a vector space and $Y$ be a non-empty compact convex subset of a Hausdorff topological vector space. Suppose that $f$ is a real-valued function on $X \times Y$ such that for each fixed $x \in X$, the map $y \mapsto f(x, y)$, i.e. $f(x, \cdot)$, is lower semi-continuous and convex on $Y$ and for each fixed $y \in Y$, the map $x \mapsto$ $f(x, y)$, i.e. $f(\cdot, y)$ is concave on $X$. Then

$$
\min _{y \in Y} \sup _{x \in X} f(x, y)=\sup _{x \in X} \min _{y \in Y} f(x, y) .
$$

The following result is Lemma 3 in [1]:

Lemma 2.4. Let $E$ be a Hausdorff topological vector space, $A \in \mathcal{F}(E), X=\operatorname{co}(A)$, and $T: X \rightarrow 2^{E^{*}}$ be upper semicontinuous from $X$ to the weak*-topology on $E^{*}$ such that $T(x)$ is weak*-compact. Let $f: X \times X \rightarrow \mathbb{R}$ be defined by $f(x, y)=\inf _{w \in T(y)} \operatorname{Re}\langle w, y-x\rangle$ for all $x, y \in X$. Then for each fixed $x \in X, y \mapsto f(x, y)$ is lower semi-continuous on $X$.

\section{Generalized Quasi-Variational Inequalities of Pseudo-Monotone Type III and Strongly Pseudo-Monotone Type III Operators}

In this section, we shall obtain some general existence theorems for the solutions to the generalized quasivariational inequalities for pseudo-monotone type III operators and strongly pseudo-monotone type III operators on non-compact sets.

We shall first establish the following result:

Theorem 3.1. Let $E$ be a locally convex Hausdorff 
topological vector space, $X$ be a non-empty paracompact convex and bounded subset of $E$ and $h: E \rightarrow \mathbb{R}$ be convex with $h(X)$ bounded. Let $S: X \rightarrow 2^{X}$ be upper semi-continuous such that each $S(x)$ is compact convex and $T: X \rightarrow 2^{E^{*}}$ be an $h$-pseudo-monotone type III (respectively, strongly h-pseudomonotone type III) operator and be upper semi-continuous from $\operatorname{co}(A)$ to the weak*-topology on $E^{*}$ for each $A \in \mathcal{F}(X)$ and $T(X)$ is strongly bounded. Also, for each $x \in X, T(x)$ is weak*-compact convex. Suppose that the set

$\Sigma=\left\{y \in X: \sup _{x \in S(y)}\left[\inf _{w \in T(y)} \operatorname{Re}\langle w, y-x\rangle+h(y)-h(x)\right]>0\right\}$

is open in $X$ and the following conditions are satisfied:

(a) for each $A \in \mathcal{F}(X)$ and each $x, y \in \operatorname{co}(A)$ and any net $\left\{y_{\alpha}\right\}_{\alpha \in \Gamma}$ in $X$ converging to $y$, we have $\lim \sup _{\alpha}\left[\inf _{u \in T\left(y_{\alpha}\right)} R e\left\langle u, y_{\alpha}-x\right\rangle+h\left(y_{\alpha}\right)-h(x)\right] \leq$ 0 whenever $\lim \sup _{\alpha}\left[\inf _{u \in T\left(y_{\alpha}\right)} \operatorname{Re}\left\langle u, y_{\alpha}-y\right\rangle+\right.$ $\left.h\left(y_{\alpha}\right)-h(y)\right] \leq 0$, and

(b) $\lim \sup _{\alpha}\left[\inf _{w \in T\left(y_{\alpha}\right)} \operatorname{Re}\left\langle w, y_{\alpha}-x\right\rangle+h\left(y_{\alpha}\right)-\right.$ $h(x)] \geq \inf _{w \in T(y)} \operatorname{Re}\langle w, y-x\rangle+h(y)-h(x)$

whenever $\quad \lim \sup _{\alpha}\left[\inf _{w \in T\left(y_{\alpha}\right)} \operatorname{Re}\left\langle w, y_{\alpha}-y\right\rangle+\right.$ $\left.h\left(y_{\alpha}\right)-h(y)\right] \geq \inf _{w \in T(y)} \operatorname{Re}\langle w, y-x\rangle+h(y)-$ $h(x)$.

Suppose further that there exists a non-empty compact subset $K$ of $X$ and a point $x_{0} \in X$ such that $x_{0} \in K \cap S(y)$ and $\inf _{w \in T(y)} R e\left\langle w, y-x_{0}\right\rangle+h(y)-h\left(x_{0}\right)>0$ for all $y \in X \backslash K$. Then there exists a point $\hat{y} \in K$ such that

(i) $\hat{y} \in S(\hat{y})$ and

(ii) there exists a point $\widehat{w} \in T(\hat{y})$ with $\operatorname{Re}\langle\widehat{w}, \hat{y}-x\rangle \leq$ $h(x)-h(\hat{y})$ for all $x \in S(\hat{y})$.

Proof. We shall complete the proof in three steps as follows:

Step 1 . There exists a point $\hat{y} \in X$ such that $\hat{y} \in S(\hat{y})$ and

$$
\sup _{x \in S(\hat{y})}\left[\inf _{w \in T(\hat{y})} \operatorname{Re}\langle w, \hat{y}-x\rangle+h(\hat{y})-h(x)\right] \leq 0 .
$$

Suppose the contrary. Then for each $y \in X$, either $y \notin$ $S(y)$ or there exists $x \in S(y)$ such that $\inf _{w \in T(y)} \operatorname{Re}\langle w, y-$ $x\rangle+h(y)-h(x)>0$; that is, for each $y \in X$, either $y \notin S(y)$ or $y \in \Sigma$. If $y \notin S(y)$, then by a separation theorem for convex sets in locally convex Hausdorff topological vector spaces, there exists $p \in E^{*}$ such that $\operatorname{Re}\langle p, y\rangle-$ $\sup _{x \in S(y)} \operatorname{Re}\langle p, x\rangle>0$. For each $y \in X$, set

$$
\gamma(y):=\sup _{x \in S(y)}\left[\inf _{w \in T(y)} \operatorname{Re}\langle w, y-x\rangle+h(y)-h(x)\right] .
$$

Let $V_{0}:=\{y \in X \mid \gamma(y)>0\}=\Sigma$ and for each $p \in E^{*}$, set

$$
V_{p}:=\left\{y \in X: \operatorname{Re}\langle p, y\rangle-\sup _{x \in S(y)} \operatorname{Re}\langle p, x\rangle>0 .\right.
$$

Then $X=V_{0} \cup \cup_{p \in E^{*}} V_{p}$. Since each $V_{p}$ is open in $X$ by Lemma 2.1 and $V_{0}$ is open in $X$ by hypothesis, $\left\{V_{0}, V_{p}: p \in\right.$ $\left.E^{*}\right\}$ is an open covering for $X$. Since $X$ is paracompact, there is a continuous partition of unity $\left\{\beta_{0}, \beta_{p}: p \in E^{*}\right\}$ for $X$ subordinated to the open cover $\left\{V_{0}, V_{p}: p \in E^{*}\right\}$ (see, for example, Theorem VIII.4.2 of Dugundji in [11]), i.e. for each $p \in E^{*}, \beta_{p}: X \rightarrow[0,1]$ and $\beta_{0}: X \rightarrow[0,1]$ are continuous functions such that for each $p \in E^{*}, \beta_{p}(y)=0$ for all $y \in X \backslash V_{p} \quad$ and $\quad \beta_{0}(y)=0$ for all $y \in X \backslash V_{0} \quad$ and $\left\{\right.$ support $\beta_{0}$, support $\left.\beta_{p}: p \in E^{*}\right\}$ is locally finite and $\beta_{0}(y)+\sum_{p \in E^{*}} \beta_{p}(y)=1$ for each $y \in X$. Note that for each $A \in \mathcal{F}(X), h$ is continuous on $\operatorname{co}(A)$ (see e.g. [12, Corollary $10.1 .1, \mathrm{p} .83])$. Define $\phi: X \times X \rightarrow \mathbb{R}$ by

$$
\begin{gathered}
\phi(x, y)=\beta_{0}(y)\left[\min _{w \in T(y)} \operatorname{Re}\langle w, y-x\rangle+h(y)-h(x)\right] \\
+\sum_{p \in E^{*}} \beta_{p}(y) \operatorname{Re}\langle p, y-x\rangle
\end{gathered}
$$

for each $x, y \in X$. Then we have the following:

(i) Since $E$ is Hausdorff, for each $A \in \mathcal{F}(X)$ and each fixed $x \in \operatorname{co}(A)$, the map

$$
y \mapsto \min _{w \in T(y)} R e\langle w, y-x\rangle+h(y)-h(x)
$$

is continuous on $\operatorname{co}(A)$ by Lemma 2.3 and the fact that $h$ is continuous on $\operatorname{co}(A)$ and therefore the map

$$
y \mapsto \beta_{0}(y)\left[\min _{w \in T(y)} R e\langle w, y-x\rangle+h(y)-h(x)\right]
$$

is lower semi-continuous on $\operatorname{co}(A)$ by Lemma 2.2. Also, for each fixed $x \in X$,

$$
y \mapsto \sum_{p \in E^{*}} \beta_{p}(y) \operatorname{Re}\langle p, y-x\rangle
$$

is continuous on $X$. Hence, for each $A \in \mathcal{F}(X)$ and each fixed $x \in \operatorname{co}(A)$, the map $y \mapsto \phi(x, y)$ is lower semi-continuous on $\operatorname{co}(A)$.

(ii) For each $A \in \mathcal{F}(X)$ and for each $y \in \operatorname{co}(A)$, $\min _{x \in A} \phi(x, y) \leq 0$. If this were false, then there exists some $A=\left\{x_{1}, \ldots, x_{n}\right\} \in \mathcal{F}(X)$ and some $y \in \operatorname{co}(A)$, say $y=\sum_{i=1}^{n} \lambda_{i} x_{i} \quad$ with $\sum_{i=1}^{n} \lambda_{i}=1$, such that $\min _{1 \leq i \leq n} \phi\left(x_{i}, y\right)>0$. Then for each $i=1, \ldots, n$,

$$
\begin{gathered}
\beta_{0}(y)\left[\min _{w \in T(y)} \operatorname{Re}\left\langle w, y-x_{i}\right\rangle+h(y)-h\left(x_{i}\right)\right]+ \\
\sum_{p \in E^{*}} \beta_{p}(y) \operatorname{Re}\left\langle p, y-x_{i}\right\rangle>0,
\end{gathered}
$$

so that

$$
\begin{aligned}
& 0=\phi(y, y)=\beta_{0}(y)\left[\min _{w \in T(y)} \operatorname{Re}\left\langle w, y-\sum_{i=1}^{n} \lambda_{i} x_{i}\right\rangle+h(y)\right. \\
&\left.-h\left(\sum_{i=1}^{n} \lambda_{i} x_{i}\right)\right] \\
&+\sum_{p \in E^{*}} \beta_{p}(y) \operatorname{Re}\left\langle p, y-\sum_{i=1}^{n} \lambda_{i} x_{i}\right\rangle \\
& \geq \sum_{i=1}^{n} \lambda_{i}\left(\beta _ { 0 } ( y ) \left[\min _{w \in T(y)} \operatorname{Re}\left\langle w, y-x_{i}\right\rangle\right.\right. \\
&\left.+h(y)-h\left(x_{i}\right)\right] \\
&\left.+\sum_{p \in E^{*}} \beta_{p}(y) \operatorname{Re}\left\langle p, y-x_{i}\right\rangle\right)>0,
\end{aligned}
$$


which is a contradiction.

(iii) Suppose that $\mathrm{A} \in \mathcal{F}(\mathrm{X})$, and $\left\{\mathrm{y}_{\alpha}\right\}_{\alpha \in \Gamma}$ is a net in $\mathrm{X}$ converging to $\mathrm{y}$ with $\phi\left(\mathrm{tx}+(1-\mathrm{t}) \mathrm{y}, \mathrm{y}_{\alpha}\right) \leq 0$ for all $\alpha \in \Gamma$ and all $\mathrm{t} \in[0,1]$.

Case 1: $\beta_{0}(y)=0$.

Since $\beta_{0}$ is continuous and $y_{\alpha} \rightarrow y$, we have $\beta_{0}\left(y_{\alpha}\right) \rightarrow$ $\beta_{0}(y)=0$. Note that $\beta_{0}\left(y_{\alpha}\right) \geq 0$ for each $\alpha \in \Gamma$ and $\beta_{0}\left(y_{\alpha}\right) \rightarrow 0$. Since $T(X)$ is strongly bounded and $\left\{y_{\alpha}\right\}_{\alpha \in \Gamma}$ is a bounded set, it follows that

$\lim \sup _{\alpha}\left[\beta_{0}\left(y_{\alpha}\right)\left[\min _{w \in T\left(y_{\alpha}\right)} \operatorname{Re}\left\langle w, y_{\alpha}-x\right\rangle+h\left(y_{\alpha}\right)-\right.\right.$

$$
h(x)]]=0 \text {. }
$$

Also, we have

$$
\beta_{0}(y)\left[\min _{w \in T(y)} R e\langle w, y-x\rangle+h(y)-h(x)\right]=0 .
$$

Thus it follows that

$$
\begin{array}{r}
\lim \sup _{\alpha}\left[\beta _ { 0 } ( y _ { \alpha } ) \left[\min _{w \in T\left(y_{\alpha}\right)} \operatorname{Re}\left\langle w, y_{\alpha}-x\right\rangle+h\left(y_{\alpha}\right)-\right.\right. \\
h(x)]]+\sum_{p \in E^{*}} \beta_{p}(y) \operatorname{Re}\langle p, y-x\rangle= \\
\beta_{0}(y)\left[\min _{w \in T(y)} \operatorname{Re}\langle w, y-x\rangle+h(y)-h(x)\right]+ \\
\sum_{p \in E^{*}} \beta_{p}(y) \operatorname{Re}\langle p, y-x\rangle .
\end{array}
$$

When $t=1$, we have $\phi\left(x, y_{\alpha}\right) \leq 0$ for all $\alpha \in \Gamma$, i.e.

$$
\begin{array}{r}
\beta_{0}\left(y_{\alpha}\right)\left[\min _{w \in T\left(y_{\alpha}\right)} \operatorname{Re}\left\langle w, y_{\alpha}-x\right\rangle+h\left(y_{\alpha}\right)-h(x)\right]+ \\
\sum_{p \in E^{*}} \beta_{p}\left(y_{\alpha}\right) \operatorname{Re}\left\langle p, y_{\alpha}-x\right\rangle \leq 0
\end{array}
$$

for all $\alpha \in \Gamma$. Therefore, by (2.3), we have

$$
\begin{aligned}
\limsup _{\alpha}\left[\beta_{0}\left(y_{\alpha}\right)\right. & {\left.\left[\min _{w \in T\left(y_{\alpha}\right)} \operatorname{Re}\left\langle w, y_{\alpha}-x\right\rangle+h\left(y_{\alpha}\right)-h(x)\right]\right] } \\
& +\liminf _{\alpha}\left[\sum_{p \in E^{*}} \beta_{p}\left(y_{\alpha}\right) \operatorname{Re}\left\langle p, y_{\alpha}-x\right\rangle\right] \\
& \leq \limsup _{\alpha}\left[\beta _ { 0 } ( y _ { \alpha } ) \left[\min _{w \in T\left(y_{\alpha}\right)} \operatorname{Re}\left\langle w, y_{\alpha}-x\right\rangle\right.\right. \\
& \left.\left.+h\left(y_{\alpha}\right)-h(x)\right]\right] \\
& +\sum_{p \in E^{*}} \beta_{p}\left(y_{\alpha}\right) \operatorname{Re}\left\langle p, y_{\alpha}-x\right\rangle \leq 0
\end{aligned}
$$

and so

$\lim \sup _{\alpha}\left[\beta_{0}\left(y_{\alpha}\right)\left[\min _{w \in T\left(y_{\alpha}\right)} \operatorname{Re}\left\langle w, y_{\alpha}-x\right\rangle+h\left(y_{\alpha}\right)-\right.\right.$

$$
h(x)]]+\sum_{p \in E^{*}} \beta_{p}(y) \operatorname{Re}\langle p, y-x\rangle \leq 0 .
$$

Hence, by (2.2) and (2.4), we have $\phi(x, y) \leq 0$.

Case 2. $\beta_{0}(y)>0$.

Since $\beta_{0}\left(y_{\alpha}\right) \rightarrow \beta_{0}(y)$, there exists $\lambda \in \Gamma$ such that $\beta_{0}\left(y_{\alpha}\right)>0$ for all $\alpha \geq \lambda$.

When $t=0$, we have $\phi\left(y, y_{\alpha}\right) \leq 0$ for all $\alpha \in \Gamma$, i.e.

$$
\beta_{0}\left(y_{\alpha}\right)\left[\min _{w \in T\left(y_{\alpha}\right)} \operatorname{Re}\left\langle w, y_{\alpha}-y\right\rangle+h\left(y_{\alpha}\right)-h(y)\right]+
$$

$$
\sum_{p \in E^{*}} \beta_{p}\left(y_{\alpha}\right) \operatorname{Re}\left\langle p, y_{\alpha}-y\right\rangle \leq 0
$$

for all $\alpha \in \Gamma$.

Thus

$\lim \sup _{\alpha}\left[\beta_{0}\left(y_{\alpha}\right)\left[\min _{w \in T\left(y_{\alpha}\right)} R e\left\langle w, y_{\alpha}-y\right\rangle+h\left(y_{\alpha}\right)-\right.\right.$

$$
\left.h(y)]+\sum_{p \in E^{*}} \beta_{p}\left(y_{\alpha}\right) \operatorname{Re}\left\langle p, y_{\alpha}-y\right\rangle\right] \leq 0 \text {. }
$$

Hence

$$
\begin{gathered}
\lim \sup _{\alpha}\left[\beta _ { 0 } ( y _ { \alpha } ) \left[\min _{w \in T\left(y_{\alpha}\right)} \operatorname{Re}\left\langle w, y_{\alpha}-y\right\rangle+h\left(y_{\alpha}\right)-\right.\right. \\
h(y)]]+\lim \inf _{\alpha}\left[\sum_{p \in E^{*}} \beta_{p}\left(y_{\alpha}\right) \operatorname{Re}\left\langle p, y_{\alpha}-y\right\rangle\right] \leq \\
\lim \sup _{\alpha}\left[\beta _ { 0 } ( y _ { \alpha } ) \left[\min _{w \in T\left(y_{\alpha}\right)} \operatorname{Re}\left\langle w, y_{\alpha}-y\right\rangle+h\left(y_{\alpha}\right)-\right.\right. \\
\left.h(y)]+\sum_{p \in E^{*}} \beta_{p}\left(y_{\alpha}\right) \operatorname{Re}\left\langle p, y_{\alpha}-y\right\rangle\right] \leq 0(\operatorname{by}(2.5)) .
\end{gathered}
$$

Since $\lim \inf _{\alpha}\left[\sum_{p \in E^{*}} \beta_{p}\left(y_{\alpha}\right) \operatorname{Re}\left\langle p, y_{\alpha}-y\right\rangle\right]=0$, we have

$$
\begin{array}{r}
\limsup \alpha\left[\beta _ { 0 } ( y _ { \alpha } ) \left[\min _{w \in T\left(y_{\alpha}\right)} \operatorname{Re}\left\langle w, y_{\alpha}-y\right\rangle+h\left(y_{\alpha}\right)-\right.\right. \\
h(y)]] \leq 0 .
\end{array}
$$

Since $\beta_{0}\left(y_{\alpha}\right)>0$ for all $\alpha \geq \lambda$, it follows that

$$
\begin{array}{r}
\beta_{0}(y) \lim \sup _{\alpha}\left[\min _{w \in T\left(y_{\alpha}\right)} \operatorname{Re}\left\langle w, y_{\alpha}-y\right\rangle+h\left(y_{\alpha}\right)-\right. \\
h(y)]=\lim \sup _{\alpha}\left[\beta _ { 0 } ( y _ { \alpha } ) \left[\min _{w \in T\left(y_{\alpha}\right)} \operatorname{Re}\left\langle w, y_{\alpha}-y\right\rangle+\right.\right. \\
\left.\left.h\left(y_{\alpha}\right)-h(y)\right]\right] .
\end{array}
$$

Since $\beta_{0}(y)>0$, by (2.6) and (2.7) we have

$$
\lim \sup _{\alpha}\left[\min _{w \in T\left(y_{\alpha}\right)} \operatorname{Re}\left\langle w, y_{\alpha}-y\right\rangle+h\left(y_{\alpha}\right)-h(y)\right] \leq 0 .
$$

Then, by hypothesis (a), we have

$$
\lim \sup _{\alpha}\left[\min _{w \in T\left(y_{\alpha}\right)} \operatorname{Re}\left\langle w, y_{\alpha}-x\right\rangle+h\left(y_{\alpha}\right)-h(x)\right] \leq 0 .
$$

Since $T$ is a pseudo-monotone type III operator, we have $\lim \sup _{\alpha}\left[\min _{w \in T\left(y_{\alpha}\right)} \operatorname{Re}\left\langle w, y_{\alpha}-y\right\rangle+h\left(y_{\alpha}\right)-h(y)\right] \geq$ $\min _{w \in T(y)} \operatorname{Re}\langle w, y-x\rangle+h(y)-h(x)$.

Then, by hypothesis (b), we have

$\lim \sup _{\alpha}\left[\min _{w \in T\left(y_{\alpha}\right)} R e\left\langle w, y_{\alpha}-x\right\rangle+h\left(y_{\alpha}\right)-h(x)\right] \geq$ $\min _{w \in T(y)} \operatorname{Re}\langle w, y-x\rangle+h(y)-h(x)$.

Since $\beta_{0}(y)>0$, we have

$$
\beta_{0}(y) \lim \sup _{\alpha}\left[\min _{w \in T\left(y_{\alpha}\right)} \operatorname{Re}\left\langle w, y_{\alpha}-x\right\rangle+h\left(y_{\alpha}\right)-\right.
$$
$h(x)] \geq \beta_{0}(y)\left[\min _{w \in T(y)} \operatorname{Re}\langle w, y-x\rangle+h(y)-h(x)\right]$ (2.8)

Thus, 


$$
\begin{aligned}
\beta_{0}(y) \lim \sup _{\alpha}\left[\min _{w \in T\left(y_{\alpha}\right)} \operatorname{Re}\left\langle w, y_{\alpha}-x\right\rangle+h\left(y_{\alpha}\right)-h(x)\right] \\
\quad+\sum_{p \in E^{*}} \beta_{p}(y) \operatorname{Re}\langle p, y \\
\quad-x\rangle \geq \beta_{0}(y)\left[\min _{w \in T(y)} \operatorname{Re}\langle w, y-x\rangle+h(y)\right. \\
\quad-h(x)]+\sum_{p \in E^{*}} \beta_{p}(y) \operatorname{Re}\langle p, y-x\rangle .
\end{aligned}
$$

Again, when $t=1$, we have $\phi\left(x, y_{\alpha}\right) \leq 0$ for all $\alpha \in \Gamma$, i.e.

$$
\begin{aligned}
\beta_{0}\left(y_{\alpha}\right)\left[\min _{w \in T\left(y_{\alpha}\right)} \operatorname{Re}\left\langle w, y_{\alpha}-x\right\rangle+h\left(y_{\alpha}\right)-h(x)\right] \\
+\sum_{p \in E^{*}} \beta_{p}\left(y_{\alpha}\right) \operatorname{Re}\left\langle p, y_{\alpha}-x\right\rangle \leq 0
\end{aligned}
$$

for all $\alpha \in \Gamma$.

Thus

$0 \geq \lim \sup _{\alpha}\left[\beta_{0}\left(y_{\alpha}\right)\left[\min _{w \in T\left(y_{\alpha}\right)} \operatorname{Re}\left\langle w, y_{\alpha}-x\right\rangle+h\left(y_{\alpha}\right)-\right.\right.$ $\left.h(x)]+\sum_{p \in E^{*}} \beta_{p}\left(y_{\alpha}\right) \operatorname{Re}\left\langle p, y_{\alpha}-x\right\rangle\right] \geq$ $\lim \sup _{\alpha}\left[\beta_{0}\left(y_{\alpha}\right)\left[\min _{w \in T\left(y_{\alpha}\right)} \operatorname{Re}\left\langle w, y_{\alpha}-x\right\rangle+h\left(y_{\alpha}\right)-\right.\right.$ $h(x)]]+\liminf _{\alpha}\left[\sum_{p \in E^{*}} \beta_{p}\left(y_{\alpha}\right) \operatorname{Re}\left\langle p, y_{\alpha}-x\right\rangle\right]=$ $\beta_{0}(y)\left[\lim \sup _{\alpha}\left[\min _{w \in T\left(y_{\alpha}\right)} \operatorname{Re}\left\langle w, y_{\alpha}-x\right\rangle+h\left(y_{\alpha}\right)-\right.\right.$ $h(x)]]+\sum_{p \in E^{*}} \beta_{p}(y) \operatorname{Re}\langle p, y-x\rangle \geq$ $\beta_{0}(y)\left[\min _{w \in T(y)} \operatorname{Re}\langle w, y-x\rangle+h(y)-h(x)\right]+$ $\sum_{p \in E^{*}} \beta_{p}(y) \operatorname{Re}\langle p, y-x\rangle$ (by (2.8)).

Hence, we have $\phi(x, y) \leq 0$.

(iv) By hypothesis, there exists a non-empty compact (and therefore closed) subset $\mathrm{K}$ of $\mathrm{X}$ and a point $\mathrm{x}_{0} \in \mathrm{X}$ such that $\mathrm{x}_{0} \in \mathrm{K} \cap \mathrm{S}(\mathrm{y})$ and $\inf _{\mathrm{w} \in \mathrm{T}(\mathrm{y})} \operatorname{Re}\left\langle\mathrm{w}, \mathrm{y}-\mathrm{x}_{0}\right\rangle+$ $\mathrm{h}(\mathrm{y})-\mathrm{h}\left(\mathrm{x}_{0}\right)>0$ for all $\mathrm{y} \in \mathrm{X} \backslash \mathrm{K}$.

Thus, for each $y \in X \backslash K, \sup _{x \in S(y)} \inf _{w \in T(y)}[\operatorname{Re}\langle w, y-$ $x\rangle+h(y)-h(x)]>0$. Hence, $\quad y \in V_{0} \quad$ and $\beta_{0}(y)\left[\inf _{w \in T(y)} \operatorname{Re}\left\langle w, y-x_{0}\right\rangle+h(y)-h\left(x_{0}\right)\right]>0$ for all $y \in X \backslash K$; also, $\operatorname{Re}\left\langle p, y-x_{0}\right\rangle>0$ whenever $\beta_{p}(y)>0$ for $p \in E^{*}$.

Consequently,

$$
\begin{gathered}
\phi\left(x_{0}, y\right)=\beta_{0}(y)\left[\inf _{w \in T(y)} \operatorname{Re}\left\langle w, y-x_{0}\right\rangle+h(y)-h\left(x_{0}\right)\right] \\
+\sum_{p \in E^{*}} \beta_{p}(y) \operatorname{Re}\left\langle p, y-x_{0}\right\rangle>0
\end{gathered}
$$

for all $y \in X \backslash K$.

Thus, the hypothesis of $(d)$ of Theorem 1.3 is satisfied trivially. (If $T$ is a strongly $h$-quasi-pseudo-monotone type III operator, we equip $E$ with the weak topology.) Thus $\phi$ satisfies all the hypotheses of Theorem 1.3. Hence, by Theorem 1.3, there exists a point $\hat{y} \in K$ such that $\phi(x, \hat{y}) \leq$ 0 for all $x \in X$, i.e.

$$
\begin{gathered}
\beta_{0}(\hat{y})\left[\min _{w \in T(\hat{y})} \operatorname{Re}\langle w, \hat{y}-x\rangle+h(\hat{y})-h(x)\right]+ \\
\sum_{p \in E^{*}} \beta_{p}(\hat{y}) \operatorname{Re}\langle p, \hat{y}-x\rangle \leq 0
\end{gathered}
$$

for all $x \in X$.

If $\beta_{0}(\hat{y})>0$, then $\hat{y} \in V_{0}=\Sigma$, so that $\gamma(\hat{y})>0$. Choose $\hat{x} \in S(\hat{y}) \subset X$ such that

$$
\inf _{w \in T(\hat{y})} \operatorname{Re}\langle w, \hat{y}-\hat{x}\rangle+h(\hat{y})-h(\hat{x}) \geq \frac{\gamma(\hat{y})}{2}>0 .
$$

Then it follows that

$$
\beta_{0}(\hat{y})\left[\inf _{w \in T(\hat{y})} \operatorname{Re}\langle w, \hat{y}-\hat{x}\rangle+h(\hat{y})-h(\hat{x})\right]>0 .
$$

If $\beta_{p}(\hat{y})>0$ for some $p \in E^{*}$, then $\hat{y} \in V_{p}$ and hence

$$
\operatorname{Re}\langle p, \hat{y}\rangle>\sup _{x \in S(\hat{y})} \operatorname{Re}\langle p, x\rangle \geq \operatorname{Re}\langle p, \hat{x}\rangle
$$

and so $\operatorname{Re}\langle p, \hat{y}-\hat{x}\rangle>0$. Then we see that $\beta_{p}(\hat{y}) \operatorname{Re}\langle p, \hat{y}-$ $\hat{x}>>0$ whenever $\beta_{p}(\hat{y})>0$ for $p \in E^{*}$. Since $\beta_{0}(\hat{y})>0$ or $\beta_{p}(\hat{y})>0$ for some $p \in E^{*}$, it follows that

$$
\begin{gathered}
\phi(\widehat{x}, \hat{y})=\beta_{0}(\hat{y})\left[\inf _{w \in T(\hat{y})} \operatorname{Re}\langle w, \hat{y}-\hat{x}\rangle+h(\hat{y})-h(\hat{x})\right]+ \\
\sum_{p \in E^{*}} \beta_{p}(\hat{y}) \operatorname{Re}\langle p, \hat{y}-\hat{x}\rangle>0,
\end{gathered}
$$

which contradicts (2.10). This contradiction proves Step 1. Hence we have shown that there exists a point $\hat{y} \in X$ such that $\hat{y} \in S(\hat{y})$ and

$$
\sup _{x \in S(\hat{y})}\left[\inf _{w \in T(\hat{y})} R e\langle w, \hat{y}-x\rangle+h(\hat{y})-h(x)\right] \leq 0 .
$$

Step 2. We need to show that there exists a point $\widehat{w} \in T(\hat{y})$ such that $\operatorname{Re}\langle\widehat{w}, \hat{y}-x\rangle+h(\hat{y})-h(x) \leq 0$ for all $x \in S(\hat{y})$.

From Step 1, we have

$\sup _{x \in S(\hat{y})}\left[\inf _{w \in T(\hat{y})} \operatorname{Re}\langle w, \hat{y}-x\rangle+h(\hat{y})-h(x)\right] \leq 0$,

where $T(\hat{y})$ is a weak*-compact convex subset of the Hausdorff topological vector space $E^{*}$ and $S(\hat{y})$ is a convex subset of $X$.

Now, we define $f: S(\hat{y}) \times T(\hat{y}) \rightarrow \mathbb{R}$ by $f(x . w)=$ $\operatorname{Re}\langle w, \hat{y}-x\rangle+h(\hat{y})-h(x)$ for each $x \in S(\hat{y})$ and $w \in$ $T(\hat{y})$. Then, for each fixed $x \in S(\hat{y})$, the mapping $w \mapsto$ $f(x, w)$ is convex and continuous on $T(\hat{y})$ and, for each fixed $w \in T(\hat{y})$, the mapping $x \mapsto f(x, w)$ is concave on $S(\hat{y})$. So, we can apply Kneser's Minimax Theorem (Theorem 2.3) and obtain the following:

$$
\begin{gathered}
\min _{w \in T(\hat{y})} \sup _{x \in S(\hat{y})}[\operatorname{Re}\langle w, \hat{y}-x\rangle+h(\hat{y})-h(x)]= \\
\sup _{x \in S(\hat{y})}\left[\min _{w \in T(\hat{y})}[\operatorname{Re}\langle w, \hat{y}-x\rangle+h(\hat{y})-h(x)]\right] .
\end{gathered}
$$

Hence, by (2.11), we obtain

$$
\min _{w \in T(\hat{y})} \sup _{x \in S(\hat{y})}[\operatorname{Re}\langle w, \hat{y}-x\rangle+h(\hat{y})-h(x)] \leq 0 .
$$

Since $T(\hat{y})$ is compact, there exists $\widehat{w} \in T(\hat{y})$ such that

$$
\operatorname{Re}\langle\widehat{w}, \hat{y}-x\rangle+h(\hat{y})-h(x) \leq 0
$$

for all $x \in S(\hat{y})$. This completes the proof.

When $X$ is compact, we obtain the following immediate consequence of Theorem 3.1: 
Theorem 3.2. Let $E$ be a locally convex Hausdorff topological vector space, $X$ be a non-empty compact convex subset of $E$ and $h: E \rightarrow \mathbb{R}$ be convex with $h(X)$ bounded. Let $S: X \rightarrow 2^{X}$ be upper semi-continuous such that each $S(x)$ is closed convex and $T: X \rightarrow 2^{E^{*}}$ be an $h$-pseudo-monotone type III (respectively, a strongly $h$-pseudo-monotone type III) operator and be upper semi-continuous from $\operatorname{co}(A)$ to the weak*-topology on $E^{*}$ for each $A \in \mathcal{F}(X)$ and $T(X)$ is strongly bounded. Also, for each $x \in X, T(x)$ is weak*compact convex. Suppose that the set

$$
\Sigma=\left\{y \in X: \sup _{x \in S(y)}\left[\inf _{w \in T(y)} \operatorname{Re}\langle w, y-x\rangle+h(y)-h(x)\right]>0\right\}
$$

is open in $X$ and the following conditions are satisfied:

(a) For each $A \in \mathcal{F}(X)$, each $x, y \in \operatorname{co}(A)$, and any net $\left\{y_{\alpha}\right\}_{\alpha \in \Gamma}$ in $X$ converging to $y$, we have

(b) $\lim \sup _{\alpha}\left[\inf _{u \in T\left(y_{\alpha}\right)} R e\left\langle u, y_{\alpha}-x\right\rangle+h\left(y_{\alpha}\right)-h(x)\right] \leq 0$, whenever $\left[u \in T\left(y_{\alpha}\right)\right.$ $\lim \sup _{\alpha}\left[\inf _{u \in T\left(y_{\alpha}\right)} \operatorname{Re}\left\langle u, y_{\alpha}-y\right\rangle+h\left(y_{\alpha}\right)-h(y)\right] \leq 0$,

(c) $\lim \sup _{\alpha}\left[\inf _{w \in T\left(y_{\alpha}\right.} \operatorname{Re}\left\langle w, y_{\alpha}-x\right\rangle+h\left(y_{\alpha}\right)-h(x)\right] \geq$ $\inf _{\ln } \operatorname{Re}\left\langle w, y \underline{y} \underline{y}_{\alpha} x_{x}\right\rangle+h(y)-h(x) \quad, \quad$ whenever $\lim _{\sup } \stackrel{(y)}{L}_{\alpha} \inf _{w \in T(y)} \operatorname{Re}\left\langle w, y_{\alpha}-y\right\rangle+h\left(y_{\alpha}\right)-h(y) \mid \geq$ $\left.\inf \operatorname{Re}\left\langle w, y \in \underline{w} \underline{y}_{\alpha}\right) x\right\rangle+h(y)-h(x)$.

Then the there exists a point $\hat{y} \in X$ such that

(i) $\hat{y} \in S(\hat{y})$ and

(ii) there exists a point $\widehat{w} \in T(\hat{y})$ with $\operatorname{Re}\langle\widehat{w}, \hat{y}-x\rangle \leq$ $h(x)-h(\hat{y})$ for all $x \in S(\hat{y})$.

Note that if the map $S: X \rightarrow 2^{X}$ is, in addition, lower semicontinuous and for each $y \in \Sigma, T$ is upper semi-continuous at $y$ in $X$, then the set $\Sigma$ in Theorem 3.1 is always open in $X$ and we obtain the following theorem:

Theorem 3.3. Let $E$ be a locally convex Hausdorff topological vector space, $X$ be a non-empty paracompact convex and bounded subset of $E$ and $h: E \rightarrow \mathbb{R}$ be convex with $h(X)$ bounded. Let $S: X \rightarrow 2^{X}$ be continuous such that each $S(x)$ is compact convex, $T: X \rightarrow 2^{E^{*}}$ be an $h$-pseudomonotone type III (respectively, strongly h-pseudo-monotone type III) operator which is upper semi-continuous from $\operatorname{co}(A)$ to the weak*-topology on $E^{*}$ for each $A \in \mathcal{F}(X)$, with $T(X)$ strongly bounded. Also, for each $x \in X, T(x)$ is weak*compact convex. Suppose that for each $y \in \Sigma=\{y \in$ $\left.X: \sup _{x \in S(y)}\left[\inf _{w \in T(y)} \operatorname{Re}\langle w, y-x\rangle+h(y)-h(x)\right]>0\right\}, T$ is upper semi-continuous at $y$ from the relative topology on $X$ to the strong topology on $E^{*}$ and the following conditions are satisfied:

(a) For each $A \in \mathcal{F}(X)$, each $x, y \in \operatorname{co}(A)$, and any net $\left\{y_{\alpha}\right\}_{\alpha \in \Gamma}$ in $X$ converging to $y$, we have $\lim \sup _{\alpha}\left[\inf _{u \in T\left(y_{\alpha}\right)} \operatorname{Re}\left\langle u, y_{\alpha}-x\right\rangle+h\left(y_{\alpha}\right)-h(x)\right] \leq 0$, whenever $\lim \sup _{\alpha}\left[\inf _{u \in T\left(y_{\alpha}\right)} R e\left\langle u, y_{\alpha}-y\right\rangle+h\left(y_{\alpha}\right)-\right.$ $h(y)] \leq 0$, and

(b) $\lim \sup _{\alpha}\left[\inf _{w \in T\left(y_{\alpha}\right)} \operatorname{Re}\left\langle w, y_{\alpha}-x\right\rangle+h\left(y_{\alpha}\right)-h(x)\right] \geq$ $\inf _{w \in T(y)} \operatorname{Re}\langle w, y-x\rangle+h(y)-h(x) \quad, \quad$ whenever

$$
\begin{aligned}
& \lim \sup _{\alpha}\left[\inf _{w \in T\left(y_{\alpha}\right)} \operatorname{Re}\left\langle w, y_{\alpha}-y\right\rangle+h\left(y_{\alpha}\right)-h(y)\right] \geq \\
& \inf _{w \in T(y)} \operatorname{Re}\langle w, y-x\rangle+h(y)-h(x) .
\end{aligned}
$$

Suppose further that there exists a non-empty compact subset $K$ of $X$ and a point $x_{0} \in X$ such that $x_{0} \in K \cap S(y)$ and $\inf _{w \in T(y)} R e\left\langle w, y-x_{0}\right\rangle+h(y)-h\left(x_{0}\right)>0$ for all $y \in X \backslash K$.

Then there exists a point $\hat{y} \in K$ such that

(i) $\hat{y} \in S(\hat{y})$ and

(ii) there exists a point $\widehat{w} \in T(\hat{y})$ with $\operatorname{Re}\langle\widehat{w}, \hat{y}-x\rangle \leq$ $h(x)-h(\hat{y})$ for all $x \in S(\hat{y})$.

The proof is similar to the proof of Theorem 3.1 in [13]. But for completeness, we shall include the detailed proof here.

Proof. The proof will follow from Theorem 3.1 if we can show that the set

$\Sigma=\left\{y \in X: \sup _{x \in S(y)}\left[\inf _{w \in T(y)} \operatorname{Re}\langle w, y-x\rangle+h(y)-h(x)\right]>0\right\}$

is open in $X$. To show that $\Sigma$ is open in $X$, we start as follows:

Let $y_{0} \in \Sigma$ be an arbitrary point. We show that there exists an open neighborhood $N_{0}$ of $y_{0}$ in $X$ such that $N_{0} \subset \Sigma$. Now, by definition of $\Sigma$, there exists a point $x_{0}$ in $S\left(y_{0}\right)$ with

$$
\inf _{w \in T\left(y_{0}\right)} R e\left\langle w, y_{0}-x_{0}\right\rangle+h\left(y_{0}\right)-h\left(x_{0}\right)>0 .
$$

Let

$$
\alpha:=\inf _{w \in T\left(y_{0}\right)} \operatorname{Re}\left\langle w, y_{0}-x_{0}\right\rangle+h\left(y_{0}\right)-h\left(x_{0}\right) .
$$

Thus, $\alpha>0$. Again, let

$$
W:=\left\{w \in E^{*}: \sup _{z_{1}, z_{2} \in X}\left|\left\langle w, z_{1}-z_{2}\right\rangle\right|<\alpha / 6\right\} .
$$

Then $W$ is a strongly open neighborhood of 0 in $E^{*}$ and so $U_{1}:=T\left(y_{0}\right)+W$ is an open neighborhood of $T\left(y_{0}\right)$ in $E^{*}$. Since $T$ is upper semi-continuous at $y_{0}$, there exists an open neighborhood $N_{1}$ of $y_{0}$ in $X$ such that $T(y) \subset U_{1}$ for all $y \in N_{1}$. Since the mapping $x \mapsto \inf _{w \in T\left(y_{0}\right)} \operatorname{Re}\left\langle w, x_{0}-x\right\rangle+$ $h\left(x_{0}\right)-h(x)$ is continuous at $x_{0}$, there exists an open neighborhood $V_{1}$ of $x_{0}$ in $X$ such that

$$
\left|\inf _{w \in T\left(y_{0}\right)} \operatorname{Re}\left\langle w, x_{0}-x\right\rangle+h\left(x_{0}\right)-h(x)\right|<\alpha / 6
$$

for all $x \in V_{1}$.

Since $x_{0} \in V_{1} \cap S\left(y_{0}\right) \neq \varnothing$ and $S$ is lower semicontinuous at $y_{0}$, there exists an open neighborhood $N_{2}$ of $y_{0}$ in $X$ such that $S(y) \cap V_{1} \neq \emptyset$ for all $y \in N_{2}$. Since the mapping $\quad y \mapsto \inf _{w \in T\left(y_{0}\right)} \operatorname{Re}\left\langle w, y-y_{0}\right\rangle+h(y)-h\left(y_{0}\right) \quad$ is continuous at $y_{0}$, there exists an open neighborhood $N_{3}$ of $y_{0}$ in $X$ such that

$$
\left|\inf _{w \in T\left(y_{0}\right)} \operatorname{Re}\left\langle w, y-y_{0}\right\rangle+h(y)-h\left(y_{0}\right)\right|<\alpha / 6
$$

for all $y \in N_{3}$.

Let $N_{0}:=N_{1} \cap N_{2} \cap N_{3}$. Then $N_{0}$ is an open neighborhood of $y_{0}$ in $X$ such that for each $y_{1} \in N_{0}$, we have the following: 
(a) $S\left(y_{1}\right) \cap V_{1} \neq \emptyset$ as $y_{1} \in N_{2}$; so, we can choose any $x_{1} \in S\left(y_{1}\right) \cap V_{1}$.

(b) $\left|\inf _{w \in T\left(y_{0}\right)} \operatorname{Re}\left\langle w, y_{1}-y_{0}\right\rangle+h\left(y_{1}\right)-h\left(y_{0}\right)\right|<\alpha / 6 \quad$ as $y_{1} \in N_{3}$.

(c) $T\left(y_{1}\right) \subset U_{1}=T\left(y_{0}\right)+W$ as $y_{1} \in N_{1}$.

(d) $\left|\inf _{w \in T\left(y_{0}\right)} \operatorname{Re}\left\langle w, x_{0}-x_{1}\right\rangle+h\left(x_{0}\right)-h\left(x_{1}\right)\right|<\alpha / 6 \quad$ as $x_{1} \in V_{1}$.

Hence, we can obtain the following by omitting the details:

$$
\begin{aligned}
& \quad \inf _{w \in T\left(y_{1}\right)} \operatorname{Re}\left\langle w, y_{1}-x_{1}\right\rangle+h\left(y_{1}\right)-h\left(x_{1}\right) \\
& \geq \inf _{w \in T\left(y_{0}\right)+W} \operatorname{Re}\left\langle w, y_{1}-x_{1}\right\rangle+h\left(y_{1}\right)-h\left(x_{1}\right) \text { by }(c) \\
& \geq \inf _{w \in T\left(y_{0}\right)} \operatorname{Re}\left\langle w, y_{1}-x_{1}\right\rangle+h\left(y_{1}\right)-h\left(x_{1}\right) \\
& \quad \quad \quad \quad \inf _{w \in W} \operatorname{Re}\left\langle w, y_{1}-x_{1}\right\rangle \\
& \geq \inf _{w \in T\left(y_{0}\right)} \operatorname{Re}\left\langle w, y_{1}-y_{0}\right\rangle+h\left(y_{1}\right)-h\left(y_{0}\right) \\
& +\inf _{w \in T\left(y_{0}\right)} \operatorname{Re}\left\langle w, y_{0}-x_{0}\right\rangle+h\left(y_{0}\right)-h\left(x_{0}\right) \\
& +\inf _{w \in T\left(y_{0}\right)} \operatorname{Re}\left\langle w, x_{0}-x_{1}\right\rangle+h\left(x_{0}\right)-h\left(x_{1}\right) \\
& +\inf _{w \in W} \operatorname{Re}\left\langle w, y_{1}-x_{1}\right\rangle \geq-\frac{\alpha}{6}+\alpha-\frac{\alpha}{6}-\frac{\alpha}{6}=\frac{\alpha}{2}>0 .
\end{aligned}
$$

Consequently, we have

$$
\sup _{x \in S\left(y_{1}\right)}\left[\inf _{w \in T\left(y_{1}\right)} \operatorname{Re}\left\langle w, y_{1}-x\right\rangle+h\left(y_{1}\right)-h(x)\right]>0
$$

since $x_{1} \in S\left(y_{1}\right)$. Hence, $y_{1} \in \Sigma$ for all $y_{1} \in N_{0}$. Therefore, $y_{0} \in N_{0} \subset \Sigma$. But $y_{0}$ was arbitrary. Consequently, $\Sigma$ is open in $X$. Thus, all the hypotheses of Theorem 3.1 are satisfied. Hence, the conclusion follows from Theorem 3.1. This completes the proof.

When $X$ is compact, we obtain the following theorem:

Theorem 3.4. Let $E$ be a locally convex Hausdorff topological vector space, $X$ be a non-empty compact convex subset of $E$ and $h: E \rightarrow \mathbb{R}$ be convex with $h(X)$ bounded. Let $S: X \rightarrow 2^{X}$ be continuous such that each $S(x)$ is closed convex, $T: X \rightarrow 2^{E^{*}}$ be an $h$-pseudo-monotone type III (respectively, strongly h-pseudo-monotone type III) operator which is upper semi-continuous from $\operatorname{co}(A)$ to the weak*topology on $E^{*}$ for each $A \in \mathcal{F}(X)$, with $T(X)$ strongly bounded. Also, for each $x \in X, T(x)$ is weak*-compact convex. Suppose that for each $y \in \Sigma=\left\{y \in X: \sup _{x \in S(y)}\left[\inf _{w \in T(y)} \operatorname{Re}\langle w, y-x\rangle+h(y)-\right.\right.$ $h(x)]>0\}, T$ is upper semi-continuous at $y$ from the relative topology on $X$ to the strong topology on $E^{*}$ and the following conditions are satisfied:

(a) For each $A \in \mathcal{F}(X)$, each $x, y \in \operatorname{co}(A)$, and any net $\left\{y_{\alpha}\right\}_{\alpha \in \Gamma}$ in $X$ converging to $y$, we have $\lim \sup _{\alpha}\left[\inf _{u \in T\left(y_{\alpha}\right)} \operatorname{Re}\left\langle u, y_{\alpha}-x\right\rangle+h\left(y_{\alpha}\right)-h(x)\right] \leq 0$ whenever $\quad \lim \sup _{\alpha}\left[\inf _{u \in T\left(y_{\alpha}\right)} \operatorname{Re}\left\langle u, y_{\alpha}-y\right\rangle+h\left(y_{\alpha}\right)-\right.$ $h(y)] \leq 0$, and

(b) $\lim \sup _{\alpha}\left[\inf _{w \in T\left(y_{\alpha}\right)} \operatorname{Re}\left\langle w, y_{\alpha}-x\right\rangle+h\left(y_{\alpha}\right)-h(x)\right] \geq$ $\inf _{w \in T(y)} \operatorname{Re}\langle w, y-x\rangle+h(y)-h(x)$ whenever

$$
\begin{aligned}
& \lim \sup _{\alpha}\left[\inf _{w \in T\left(y_{\alpha}\right)} \operatorname{Re}\left\langle w, y_{\alpha}-y\right\rangle+h\left(y_{\alpha}\right)-h(y)\right] \geq \\
& \inf _{w \in T(y)} \operatorname{Re}\langle w, y-x\rangle+h(y)-h(x) .
\end{aligned}
$$

Then there exists a point $\hat{y} \in X$ such that

(i) $\hat{y} \in S(\hat{y})$ and

(ii) there exists a point $\widehat{w} \in T(\hat{y})$ with $\operatorname{Re}\langle\widehat{w}, \hat{y}-x\rangle \leq$ $h(x)-h(\hat{y})$ for all $x \in S(\hat{y})$.

Remark 3.5. (1) Theorems 3.1, 3.2, 3.3 and 3.4 of this paper are further extensions of the results obtained in [4] on generalized quasi-variational inequalities of pseudomonotone type III and strongly pseudo-monotone type III operators.

(2) In 1985, Shih and Tan ([4]) obtained results on generalized quasi-variational inequalities in locally convex topological vector spaces and their results were obtained on compact sets where the set-valued mappings were either lower semi-continuous or upper semi-continuous. Our present paper is another extension of the original work in [4] using pseudo-monotone type III and strongly pseudomonotone type III operators on non-compact sets.

\section{Acknowledgement}

The second author was supported by the Basic Science Research Program through the National Research Foundation of Korea (NRF) funded by the Ministry of Science, ICT and Future Planning (2014R1A2A2A01002100).

\section{References}

[1] Mohammad S. R. Chowdhury and K.-K. Tan, Generalization of Ky Fan's minimax inequality with applications to generalized variational inequalities for pseudo-monotone operators and fixed point theorems, J. Math. Anal. Appl. 204 (1996), 910-929.

[2] K. Fan, A minimax inequality and applications, in "Inequalities, III" (O. Shisha, Ed.), pp.103-113, Academic Press, San Diego, 1972.

[3] D. Chan and J. S. Pang, The generalized quasi-variational inequality problem, Math. Oper. Res. 7(1982), 211-222.

[4] M.-H. Shih and K.-K. Tan, Generalized quasivariational inequalities in locally convex topological vector spaces, J. Math. Anal. Appl., 108 (1985), 333-343.

[5] Mohammad S. R. Chowdhury and E. Tarafdar, Hemicontinuous operators and some applications, Acta Math. Hungar. 83(3) (1999), 251-261.

[6] Mohammad S. R. Chowdhury, The surjectivity of upper-hemicontinuous and pseudo-monotone type II operators in reflexive Banach Spaces, Ganit: J. Bangladesh Math. Soc. 20 (2000), 45-53.

[7] W. Takahashi, Nonlinear variational inequalities and fixed point theorems, Journal of the Mathematical Society of Japan, 28 (1976), 168-181.

[8] M.-H. Shih and K.-K. Tan, Generalized bi-quasi-variational inequalities, J. Math. Anal. Appl., 143 (1989), 66-85. 
[9] H. Kneser, Sur un théorème fondamental de la théorie des jeux, C. R. Acad. Sci. Paris, 234 (1952), 2418-2420.

[10] J. P. Aubin, Applied Functional Analysis, Wiley-Interscience, New York, 1979.

[11] J. Dugundji, Topology, Allyn and Bacon, Inc., Boston, 1966.
[12] R. T. Rockafeller, Convex Analysis, Princeton Univ., Princeton, 1970.

[13] Mohammad S. R. Chowdhury and Kok-Keong Tan, Applications of pseudo-monotone operators with some kind of upper semicontinuity in generalized quasi-variational inequalities on non-compact sets, Proc. Amer. Math. Soc. 3(10) (1998), 2957-2968. 